\title{
Composition Variations in Fast Solar Wind Streams
}

\author{
R. von Steiger ${ }^{\mathrm{a}, \mathrm{b}}$, L. A. Fisk ${ }^{\mathrm{a}}$, G. Gloeckler ${ }^{\mathrm{a}, \mathrm{c}}$, N. A. Schwadron ${ }^{\mathrm{a}}$, \\ and T. H. Zurbuchen ${ }^{\mathrm{a}}$ \\ ${ }^{a}$ Department of Atmospheric, Oceanic and Space Sciences, University of Michigan, Ann Arbor, USA \\ ${ }^{b}$ International Space Science Institute, Bern, Switzerland \\ ${ }^{c}$ Department of Physics, University of Maryland, College Park, USA
}

\begin{abstract}
The Ulysses spacecraft has now completed its first revolution around the Sun on its nearly-polar orbit. Thereby it has traversed the extended high speed streams from the polar coronal holes (south in 1993/94, north in 1995/96) which were well-developed during that time of close to minimal solar activity.

It is evident that the fluctuations of both the kinetic and the compositional parameters are much weaker in the high-speed streams than they are in the slow solar wind, leading Bame to use the term "structure-free" for describing it. It was only the extended time periods Ulysses spent in the polar streams that led to the detection of some structure, the microstreams. From remote observations of the Sun it is clear that the high latitude corona is quite unstructured. The most remarkable features are the polar plumes, which are well detectable because of their higher density and brightness. Also, they are characterized by a difference in composition relative to the coronal hole plasma. These features should in principle be observable in interplanetary space, e. g. by the SWICS mass spectrometer, in the form of abundance variations of heavy ions as well as variations in their charge state composition, which serves as a proxy for the coronal temperature at the site where the stream originated. Using the unique data set of SWICS we examine to what extent polar plumes contribute to fast, coronal hole associated wind. We also study the possible connection between microstreams and polar plumes.
\end{abstract}

\section{MOTIVATION}

When in the 1970s a new, fast type of stationary solar wind was detected, Bame et al. (1) used the term "structure-free" for describing its main difference as opposed to the highly variable, slow solar wind normally encountered near the ecliptic plane. The coronal holes, from where this wind was identified to emanate (6), are themselves largely structure-free as compared to the coronal streamer belt. The title of this paper might therefore seem odd and the search for structure in the unstructured futile.

Nevertheless, careful analysis has revealed residual, yet significant, structures both in the fast streams and in coronal holes:

- Helios found residues of the supergranulation structure at $>0.3 \mathrm{AU}$, but these faded away by the time the wind had reached $1 \mathrm{AU}$ (11). More recently, in the extensive data set obtained by Ulysses in the large fast streams from the polar coronal holes at solar minimum, pressurebalanced structures and microstreams were found and characterised in the plasma and field data $(7,9)$.

- Optical observations in the EUV revealed the presence of bright, essentially vertical structures amidst the coronal holes - the polar plumes. These were reported earlier to show anomalies in their elemental composition, e. g. to have a strongly depressed $\mathrm{Ne} / \mathrm{Mg}$ ratio with respect to the solar and solar wind value (13), although this result may not be universal but only apply to the particularly bright plumes analysed in this study (2).

Composition studies in the Ulysses fast streams data have not revealed any obvious variations in the charge state and element abundance ratios at the level of detail the data have been investigated so far, and from this it was concluded that polar plumes are unlikely to be a significant source of fast solar wind (12). However, given the existence of microstreams and their possible association with polar plumes (9), it seems worthwhile to investigate them in more detail and to specifically search for any abundance variations of the kind as they were reported for polar plumes. Due to the expected weakness of the possible signals, a superposed epoch technique seems to be the tool of choice here.

\section{OBSERVATIONS AND DATA ANALYSIS}

The data used in this study were obtained by the SWICS sensor (4) on the Ulysses spacecraft. We concentrated on the two extended, $\sim 500$-day long periods the spacecraft spent in the fast streams from the polar coronal 


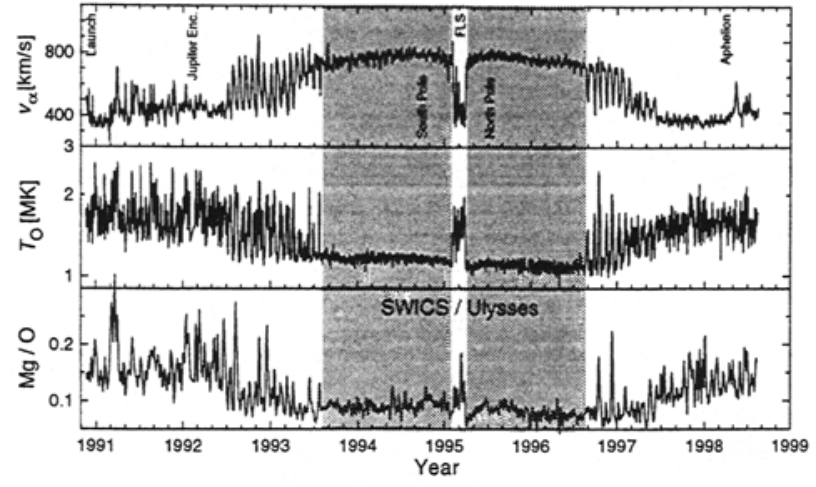

FIGURE 1. Overview of three solar wind parameters obtained with SWICS/Ulysses, alpha particle speed (top), oxygen freezing-in temperature (middle), and $\mathrm{Mg} / \mathrm{O}$ abundance ratio. The shaded regions indicate the fast streams from the polar coronal holes analysed here.

holes, as shaded in Fig. 1. The figure displays three of the most important parameters obtained by SWICS/Ulysses at a time resolution of one day. It illustrates the fundamental differences between the solar wind from the coronal holes as compared to the wind from the streamer belt region: It is faster and less variable in speed, its freezingin temperature is cooler ${ }^{1}$ and less variable, and its composition is only weakly enriched in elements with a low first ionisation potential (FIP) such as Mg and Fe. The fact that these three parameters behave in unison is one of the principal arguments for the view that the two quasistationary types of solar wind are fundamentally different in their properties and thus also in their generation and acceleration histories (3).

In order to search for structure in the time periods shaded in Fig. 1 we first ran our standard data analysis procedure of SWICS/Ulysses at a fixed time resolution of 6 hours, obtaining density, bulk velocity, and thermal speed of some 30 heavy ion species. Many of the less abundant charge states cannot be determined with statistical significance at this relatively high time resolution, but the statistical uncertainly for element abundances, which are the sum over several charge states, are of the order of less than $10 \%$ for $\mathrm{C}$ and $\mathrm{O}$ and less than $20 \%$ for the other species.

We then search for peaks and dips in the velocity of alpha particles, which is one of the most reliably determined parameters, using three different, independent methods:

Visual inspection: This is probably the most accurate and reliable method, but also the most tedious one. It

\footnotetext{
${ }^{1}$ Not to be confused with the kinetic temperature or thermal speed, which is hotter in the fast solar wind.
}

was used in (9), and the zero epoch times of 48 velocity peaks and 25 velocity dips so determined were kindly made available to us (8). However, they are not evenly distributed over the $\sim 1000$ days indicated in Fig. 1, but concentrated to the time period around the south polar pass analysed in (9) plus a relatively small number of events from the north. These peaks and dips have an average amplitude of $30 \mathrm{~km} / \mathrm{s}$ and an average half width at half maximum of 0.4 days (9).

Monotonicity: We have attempted to automate the visual method in two ways. The first one searches the data (i. e. the 6-hour alpha speed) for relative maxima (minima) which qualified as peaks (dips) if the five points to either side formed a monotonously decreasing (increasing) sequence, thereby forming a 11-point, 2.5-day structure. Since this is a rather stringent criterium, one exception per peak (dip) was allowed in the monotonicity, though. This way, 72 peaks and 71 dips were identified, with an average profile whose parameters very closely match the ones of the hand-picked peaks and dips above.

Microstream transform: Since the identification scheme above is rather stringent, we have devised a second, independent scheme as follows: First, a model peak $\mathbf{P}$ (dip D) of $2 k+1$ points is defined, which is then compared to each $(2 k+1)$-point segment of the time series $\mathbf{S}$; the midpoint of such a segment qualifies as a peak (dip) if the sum of the squared differences between the model peak and the segment falls below a certain predefined threshold:

$$
\tilde{P}_{i}=\frac{1}{2 k} \sum_{j=-k}^{k}\left(S_{i+j}-P_{j}-\langle S-P\rangle_{i}\right)^{2}<\varepsilon
$$

where the average $\langle S-P\rangle_{i}$ runs over the same $(2 \mathrm{k}+1)$ points around $i$. We call $\tilde{\mathbf{P}}$ the "microstream peak transform" and $\tilde{\mathbf{D}}$ (defined analogously) the "microstream dip transform" of $S$ since it bears some similarity to a wavelet transform except that it does not use a complete orthogonal set of wavelets, but only a single predefined model peak (dip) instead. As above, we choose $k=5$ and define as our model peak (dip) an (inverted) Gaussian of amplitude $30 \mathrm{~km} / \mathrm{s}$ and HWHM $=0.4 \mathrm{~d}$ (i. e. $\sigma=0.44 \mathrm{~d}$ ). Selecting a threshold value of $\varepsilon=7$, we find 76 peaks (i.e. $\tilde{\mathbf{P}}$ has 76 local minima below this value) and 85 dips. Not surprisingly, the parameters of the average profiles, given in Fig. 2, again match the ones of the hand-picked peaks and dips very closely.

The three methods all yield different sets of peaks and dips. Some of them are present in two or three of the sets, but this applies only to the minority. We have therefore performed the superposed epoch analysis for all three sets separately, but concentrate on the third one in this paper. The other two methods yield very much the same results, but less significantly so due to their smaller size. 


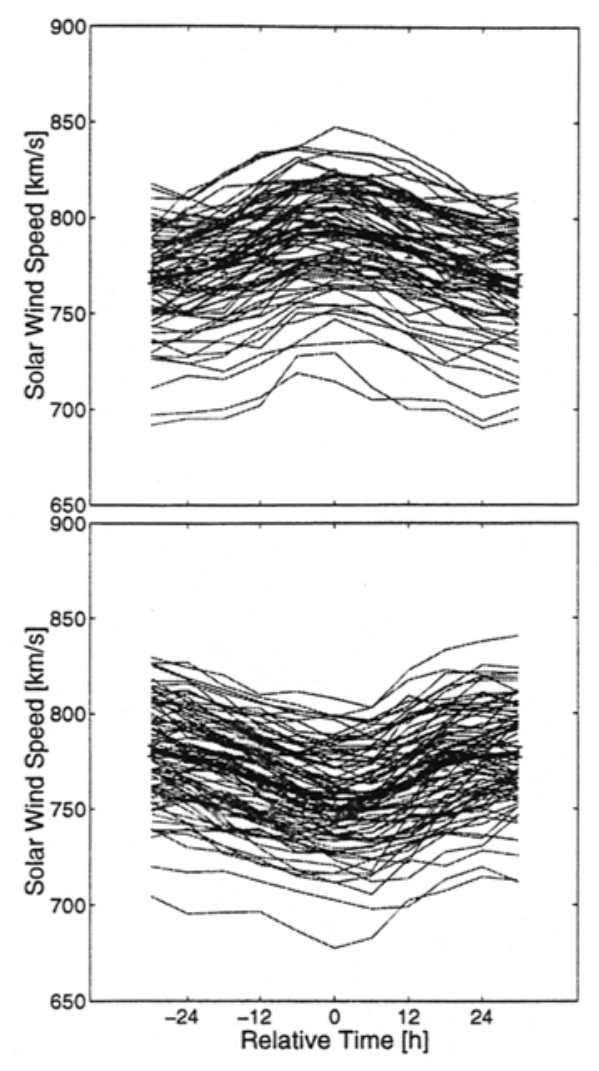

FIGURE 2. Superposed peaks (top) and dips (bottom) profile of the alpha particle speed. Given are the average (solid), median (dashed), and individual peaks/dips (gray).

\section{RESULTS AND DISCUSSION}

In Fig. 3 we give the average profiles of the $\mathrm{Ne} / \mathrm{Mg}$ and the $\mathrm{Fe} / \mathrm{O}$ abundance ratios; the error bars indicate the $1-\sigma$ standard error of the mean at each epoch. Both ratios are between an element with a low FIP (Mg, $\mathrm{Fe})$ and another with a high FIP ( $\mathrm{Ne}, \mathrm{O}$ ), which are known to vary by a factor of two between the average fast and slow solar wind types. However, there is obviously no significant variation in these ratios across the velocity peaks or dips, and the peak or dip values do not differ from the ambient fast solar wind. More specifically, the $\mathrm{Ne} / \mathrm{Mg}$ ratio never approaches the very low value of $\sim 1 / 3$ of the optical observation of a polar plume (13). The absence of a signal in the $\mathrm{Fe} / \mathrm{O}$ ratio (and in $\mathrm{Si} / \mathrm{O}$, not shown) confirms the view that there are no abundance variations across velocity peaks and dips.

In Fig. 4 we give the average profiles of the freezingin temperatures of $\mathrm{C}^{6+} / \mathrm{C}^{5+}, T_{\mathrm{C} 65}$, and of $\mathrm{C}^{5+} / \mathrm{C}^{4+}, T_{\mathrm{C} 54}$, with error bars as above. We have chosen these parameters for display because the more often used freezingin temperature $T_{\mathrm{O} 76}$ can rarely be determined with good statistics in a 6-hour time interval due to the very low

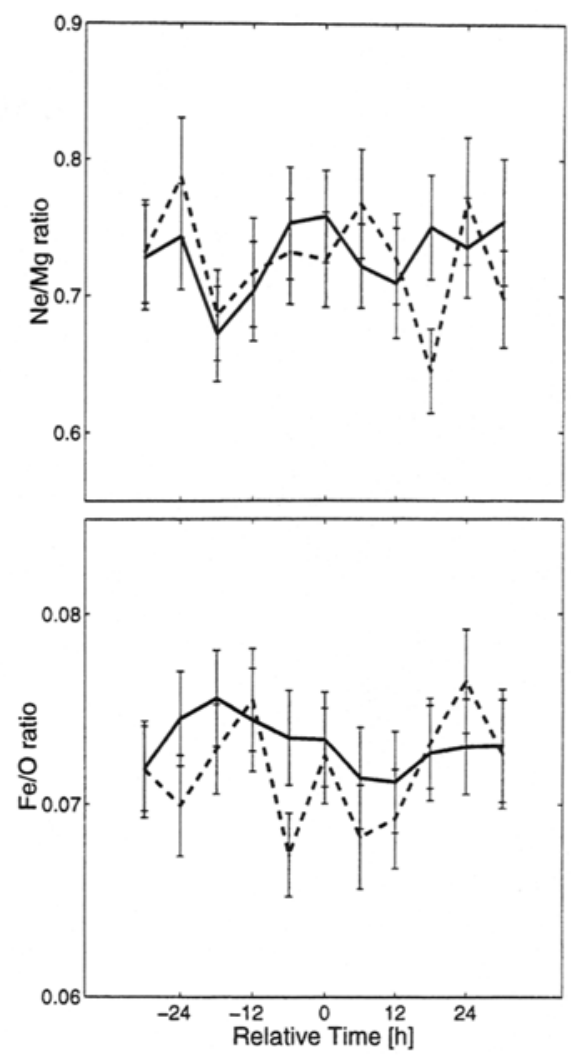

FIGURE 3. Superposed profiles of $\mathrm{Ne} / \mathrm{Mg}$ and $\mathrm{Fe} / \mathrm{O}$ abundance ratios at velocity peaks (solid line) and dips (dashed line).

abundance of $\mathrm{O}^{7+}$ in fast streams whereas the carbon charge states occur at more comparable amounts there. It is hard to assess a clear signal in Fig. 4, but there seems to be a slight anticorrelation of both temperatures with speed: velocity peaks are some $20^{\prime} 000 \mathrm{~K}$ cooler at their center than dips are. This might seem as expected, reflecting the well-known fact that the fast wind is associated with cooler freezing-in temperatures than the slow wind is. However, the result is at variance with the weak positive correlation of $T_{\mathrm{C} 65}$ and $T_{076}$ with speed when restricted to the fast wind (see Fig. 5). The weakness of the signal together with the unclear interpretation makes it questionable if there is indeed a significant change of freezing-in temperatures across velocity peaks and dips.

\section{CONCLUSIONS}

We have not found any significant deviations in abundance and charge state ratios in velocity peaks and dips from the fast, coronal hole associated solar wind surrounding them, with the possible exception of a weak anticorrelation to the freezing-in temperatures. Clearly, no sign of a strong depletion of the $\mathrm{Ne} / \mathrm{Mg}$ abundance could 

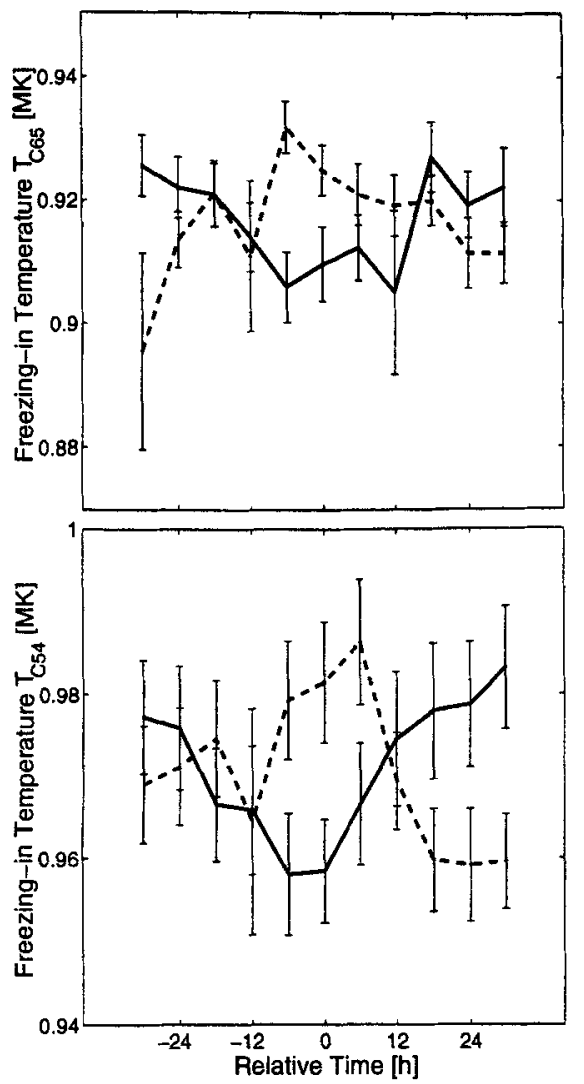

FIGURE 4. Superposed profiles of carbon freezing-in temperatures at velocity peaks (solid line) and dips (dashed line).

be found. We conclude that neither peaks nor dips are associated with polar plumes; more likely these are dynamic structures generated by processes that do not operate on the composition.

This supports the view that polar plumes are essentially static (yet transient) features with an upward flow that is slower than in the ambient coronal hole (5), thus not significantly contributing to the fast wind. If plumes are indeed anomalously composed this result also favours the picture that their contribution gets fully dissolved in the ambient fast solar wind already close to the Sun, as suggested by recent modelling (10).

\section{REFERENCES}

1. Bame, S. J., Asbridge, J. R., Feldman, W. C., and Gosling, J. T., J. Geophys. Res. 82, 1487-1492 (1977).

2. Feldman, U., personal communication (1998).

3. Geiss, J., Gloeckler, G., von Steiger, R., Balsiger, H., Fisk, L. A., Galvin, A. B., Ipavich, F. M., Livi, S., McKenzie, J. F., Ogilvie, K. W., and Wilken, B., Science 268, 10331036 (1995).

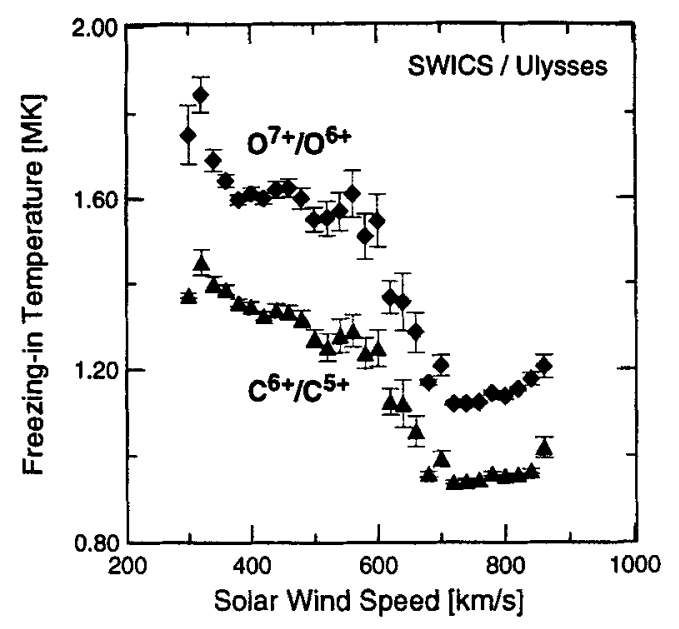

FIGURE 5. Average freezing-in temperatures $T_{\mathrm{O} 76}$ and $T_{\mathrm{C} 65}$ as a function of solar wind speed, from daily values over the complete Ulysses mission (launch to mid-1998). Note that the general anticorrelation turns into a weak positive correlation within the fast wind above $700 \mathrm{~km} / \mathrm{s}$.

4. Gloeckler, G., Geiss, J., Balsiger, H., Bedini, P., Cain, J. C., Fischer, J., Fisk, L. A., Galvin, A. B., Gliem, F., Hamilton, D. C., Hollweg, J. V., Ipavich, F. M., Joos, R., Livi, S., Lundgren, R., Mall, U., McKenzie, J. F., Ogilvie, K. W., Ottens, F., Rieck, W., Tums, E. O., von Steiger, R., Weiss, W., and Wilken, B., Astron. Astrophys. Suppl. 92, 267-289 (1992).

5. Hassler, D. M., Dammasch, I. E., Lemaire, P., Brekke, P., Curdt, W., Mason, H. E., Vial, J.-C., and Wilhelm, K., these proceedings (1998).

6. Krieger, A. S., Timothy, A. F., and Roelof, E. C., Solar Phys. 29, 505-525 (1973).

7. McComas, D. J., Barraclough, B. L., Gosling, J. T., Hammond, C. M., Neugebauer, M., Balogh, A., and Forsyth, R., J. Geophys. Res. 100, 19,893-19,902 (1995).

8. Neugebauer, M., personal communication (1998).

9. Neugebauer, M., Goldstein, B. E., McComas, D. J., Suess, S. T., and Balogh, A., J. Geophys. Res. 100, 23,389-23,395 (1995).

10. Suess, S. T., Adv. Space Res., submitted (1998).

11. Thieme, K. M., Marsch, E., and Schwenn, R., Ann. Geophys. 8, 713-723 (1990).

12. von Steiger, R., Geiss, J., and Gloeckler, G., in Cosmic Winds and the Heliosphere, edited by J. R. Jokipii, C. P. Sonett, and M. S. Giampapa, Tucson: The University of Arizona Press, 1997, pp. 581-616.

13. Widing, K. G., and Feldman, U., in Solar Wind Seven, edited by E. Marsch, and R. Schwenn, COSPAR Colloquia Series, Vol. 3, Goslar, Germany: Pergamon Press, 1992, pp. $405-410$. 